\title{
On Zwickyism
}

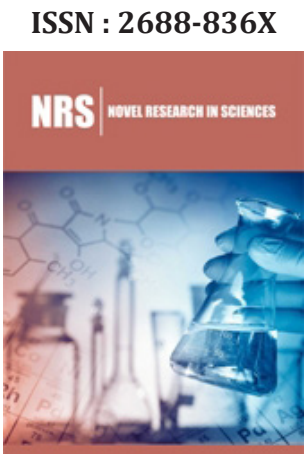

*Corresponding author: Otto E Rossler, Faculty of Science, University of Tuebingen, Auf der Morgenstelle 8, 72076 Tuebingen, Germany

Submission: 侮 September 19, 2019

Published: 侮 September 23, 2019

Volume 2 - Issue 1

How to cite this article: Otto E Rossler. On Zwickyism. Nov Res Sci.2(1). NRS.000530.2019.

DOI: 10.31031/NRS.2019.2.000530

Copyright@ Otto E Rossler, This article is distributed under the terms of the Creative Commons Attribution 4.0 International License, which permits unrestricted use and redistribution provided that the original author and source are credited.

\section{Otto E Rossler*}

Faculty of Science, University of Tuebingen, Auf der Morgenstelle 8, Germany

\section{Introduction}

A confirmation and revival of Fritz Zwicky's 1929 paper in which he explained the cosmological redshift without cosmic expansion is offered. The confirmation is based on new numerical and geometric results found by the Tuebingen school.

September 19, 2019 Swiss astronomer Fritz Zwicky discovered in 1929 that light loses energy while travelling the cosmos even in the absence of cosmic expansion. The freshly discovered cosmological redshift of Hubble was shown to be caused by gravitational interaction between the light emitters and the gravitation centers encountered along the way. Zwicky thereby glimpsed the recent new fundamental science of Cryodynamics, twin to deterministic Thermodynamics, discovered only almost 90 years later. However, the scientific community is unable to follow Zwicky's postulate-free explanation up to this day, preferring instead the postulates-laden theory of cosmic expansion. In the year 2012, the superiority of Zwicky's theory over the Big Bang postulate was demonstrated to follow from the discovery of Cryodynamics. This fact notwithstanding, the acceptance of the Big Bang postulate remains an element of the globe-wide high-school curriculum as a dogma.

The topic of "cosmic expansion" does likewise stem from the year 1929 (with a forerunner 2 years before). The famous Hubble law of cosmological redshift had just been discovered by Edwin Hubble. Zwicky's explanation was a hypothesis-free first-principles account. Nevertheless, the hypothesis of cosmic expansion based on several ad-hoc postulates has won the tug-of-war for visibility adopted for students of all ages up to this day.

A proof that Zwicky was right after all was provided almost a decade ago by the mentioned discovery of Cryodynmics as a new fundamental-physics discipline having the same rank as its famous sister, deterministic Thermodynamics. The newfound science applies to gases made up from mutually attractive rather than repulsive particles. All previous multi-particle theories had pertained to the ultimately repulsive case.

Cryodynamics implies that heavy point particles rich in kinetic energy, and by comparison very-low-energy particles like cosmic rays and photons, do not equilibrate but do rather "antiequilibrate" (that is, do equilibrate under negative time). An example for this new kind of behavior is the cosmos at large. Zwicky spotted this fact right after the discovery of the Hubble law, the famous linear distance-redshift relation valid in the cosmos.

The traditional "non-parsimonious" explanation of the Hubble law by the postulate of a global expansion has the asset of its being easy to visualize: One needs only imagine the 2-dimensional analog of the surface of a balloon that is getting inflated more and more while the structures painted on it (galaxies and their clusters) are somehow spared the fate of growing along (an inconsistency that is rarely pointed out). Then a distance-proportional redshift like that discovered empirically by Hubble follows suit as is well known.

The competing model was spotted by Zwicky: the gas-theoretic explanation already mentioned. Here, it is the attractive interaction between the particles that is responsible for the observed distance-proportional loss of the lighter particles' energy. This "dynamical interaction via Newtonian attraction" is less easy to visualize but has the asset of it doing without a single axiom having to be introduced. Zwicky was the first to conceive of it in his mind. 
Zwicky got immediately reprimanded for a minor error in his paper by the reigning doyen in the field, Sir Arthur Eddington. Although he immediately repaired the minor blunder in his next publication, he had no chance to restore his image as a brilliant newcomer.

The conceptually new mechanism offered by Zwicky did without any added postulate but required a novel thinking effort. This made it less convincing at first sight than the straightforwardlooking added axiom of cosmic expansion with its metaphysical problems (the assumption of an act of creation manifest within creation). Despite the heavy price tag of several ad-hoc axioms being attached to the expansion postulate, it won the public acceptance for 9 decades.

As everyone knows, the expansion postulate eventually found the approval of Einstein himself in 1932 after much hesitation, so that the foundations for the cosmology of the next 8 decades were effectively laid down. A struggle between Einstein and his two decades younger friend Fritz Zwicky who used to visit him at least once a year never flared up owing to Zwicky's personal modesty. Nevertheless, Zwicky would eventually win the silent struggle as the belated discovery of Cryodynamics is proof of. The latter theory seemingly represents a catastrophe for cosmology as we know it and for general relativity in the form it is known today.

Therefore, a new technical term - Zwickyism - can justifiably be introduced to date to bestow an intuitive name on the simple idea of a gas of mutually attractive rather than repulsive particles and the new features of this return to Newton's cosmology.

There exists a theme park game sometimes offered by a traveling circus in which the visitors are invited to sit individually in a kind of giant cup, many of which are moving around in a randomappearing motion during the next round paid for by the users. For it is great fun for young and old to get disoriented in a non-dangerous setting. With this picture in mind, it was quite natural to come up with the following question in conversation with Ramis Movassagh 15 years ago in Switzerland: How will a ball behave that is running across this field of moving troughs if we assume lack of friction? The answer appeared obvious: "loss of kinetic energy." But then, the opposite phenomenon would have to occur under time reversal: back up to the initial situation, from which point onwards, again a loss of energy would apply in the new direction of time: A very enlightening mental setting.

A number of papers arose from that intuitive insight, some written jointly with Ramis, starting in 2005. The topic is not without its own intrinsic implausibility, however, for an empirical reason: Namely, the many multi-particle celestial-mechanics computer simulations that have been performed over the decades. They never revealed anything like the just predicted "braking effect" for passing light and/or cosmic-ray particles. The vast number of super-computers based cosmological simulations has never revealed any systematic trend for dynamical friction. But if the above picture is correct, so that the described "systematic loss of light energy in traversing the cauldron of the moving galaxies in the cosmos" holds true, then the existence of a kind of "numerical conspiracy" in all those simulations needs to be postulated. This is a serious reason for the reader to distrust the above-described revival of Zwicky 1929.

Thus, not only the whole cosmology as we know it, but also the plethora of cosmological computer simulations performed up until now (to the extent that they are based on deterministic equations) are flatly opposed to the above new picture asserting that dynamical friction plays a major role in the cosmos. Hence Zwickyism looks like an idealistic dream that strikingly contradicts cosmological reality as it is accepted for 9 decades in the scientific literature.

Surprisingly, though, there exists one numerical study which shows that under a maximally high accuracy chosen (a so-called fourth order Verlet algorithm), there indeed is no tendency towards equipartition observable in a three-particle system subject to mutual gravitational attraction (Klaus Sonnleitner's German-language dissertation of 2010). So, the possibility arises in earnest that a common flaw characterizes the many multi-particle deterministic dynamical simulations underlying cosmological computer models. The current picture could then be described as being marred by an overlooked numerical artifact.

It goes without saying that virtually no one will be ready to believe in the present "meta-analysis" (if such a noble term is applicable). Imagine: All of humankind being allegedly trapped in a numerical artifact? Inconceivable! Actually though, it is not the fault of the computers for once but rather that of the whole scientific community which has accepted an unnecessarily complex and therefore false model of the universe in contradistinction to Zwicky's axiom-free understanding.

At this point, the reader is no doubt about to protest: A single high-accuracy computer simulation, performed 9 years ago by one scientist (Klaus Sonnleitner) cannot possibly be made the arbiter over 90 years of cosmology! This statement looks convincing. Therefore, it is important to stress here that a whole new fundamental-physics discipline called Cryodynamics, sister of deterministic Thermodynamics, has since been found after 2010 (the year of Sonnleitner's dissertation). Cryodynamics implies that a particle traversing a cauldron of randomly moving gravitation centers indeed gets reduced in its kinetic energy. For the fundamental laws of Cryodynamics happen to be precisely those of Thermodynamics - yet valid under time reversal. See the published paper titled Rolling ball in breathing plane-tree alley paradigm from 2013 which contains the geometric proof. Doyen Yakov Sinai, whose famous pure-mathematics chaos theory remains valid under time reversal in the present case of attractive potentials, has uttered no dissatisfaction towards the present author's theses over the years.

To conclude, an absolutely improbable situation in the history of science was described: Ninety years of a collective blunder- a mental tragedy. A single mathematician's doctoral dissertation, finished in 2010 (https://docplayer.org/12694730-Dissertationvorgelegt-von-dipl-math-klaus-sonnleitner-aus-erftstadtfrauenthal-bei-koeln.html), brought the first numerical proof. The first algebraic proof by Ramis Movassagh came simultaneously. A 
comprehensive dynamical proof followed suit in the form of the newfound fundamental science of Cryodynamics. All of this clearly sounds almost miraculous: Nine decades of a planet-wide wrong path trodden including a ten years long lemmings' road? Come on! These objections notwithstanding, the present account represents one of the most unlikely if true developments in the history of science. If you, the reader, become the first to confirm the geometric proof contained in the plane-tree alley paper, your feat will be amongst the notable achievements in the history of science. For up until the day of a first independent confirmation, no one is ready to believe a revolutionary fundamental result even if the latter can save all life.

(Paper presented in abridged form at the "Stefan Müller Conference" held at Dortmund on September 14, to celebrate Stefan's 70th birthday. For J.O.R.)

For possible submissions Click below: 